\title{
Limb-girdle muscular dystrophy with severe heart failure overlapping with lipodystrophy in a patient with $L M N A$ mutation p.Ser334del
}

\author{
Agnieszka Madej-Pilarczyk ${ }^{1}$ - Adam Niezgoda ${ }^{2} \cdot$ Magdalena Janus $^{3}$. \\ Romuald Wojnicz $^{4}$ - Michal Marchel ${ }^{5}$ - Anna Fidziańska ${ }^{1}$ • Stefan Grajek ${ }^{3}$. \\ Irena Hausmanowa-Petrusewicz ${ }^{1}$
}

Received: 19 July 2016 / Accepted: 22 August 2016/Published online: 1 September 2016

(C) The Author(s) 2016. This article is published with open access at Springerlink.com

\begin{abstract}
Laminopathies, a group of heterogeneous disorders associated with lamin $\mathrm{A} / \mathrm{C}$ gene (LMNA) mutations, encompass a wide spectrum of clinical phenotypes, which may present as separate disease or as overlapping syndromes. We describe a 35-year-old female in whom a novel sporadic heterozygous mutation c.1001_1003delGCC (p.Ser334del) of the LMNA gene was found. The patient presented with overlapping syndrome of heart failure secondary to dilated cardiomyopathy, limb-girdle dystrophy and partial lipodystrophy. Endomyocardial biopsy revealed strong up-regulation of HLA classes I and II antigens on microvessels and induction of the class I antigens on cardiomyocytes. On muscle biopsy, a wide range of fiber sizes and small clusters of inflammatory infiltrations were found. In the rapid progression of heart failure with arrhythmias or conduction defect, accompanied with muscle atrophy and lipodystrophy, the genetic disease should
\end{abstract}

Communicated by: Michal Witt

Anna Fidziańska and Irena Hausmanowa-Petrusewicz are deceased.

Agnieszka Madej-Pilarczyk

agamadpil@gmail.com

1 Neuromuscular Unit, Mossakowski Medical Research Centre, Polish Academy of Sciences, Pawinskiego St. 5, 02-106 Warsaw, Poland

2 Department of Neurology, Poznan University of Medical Sciences, Poznan, Poland

3 1st Department of Cardiology, Poznan University of Medical Sciences, Poznan, Poland

4 Department of Histology, School of Medicine with the Division of Dentistry, Medical University of Silesia in Katowice, Zabrze, Poland

5 1st Department of Cardiology, Medical University of Warsaw, Banacha 1a, Warsaw, Poland be taken into consideration. In addition, undefined inflammatory response and fibrosis in the heart or skeletal muscle might further justify screening of the lamin $\mathrm{A} / \mathrm{C}$ gene.

Keywords $L M N A$ gene $\cdot$ Lamin A/C $\cdot$ Laminopathy Limb-girdle muscular dystrophy $\cdot$ Cardiomyopathy

\section{Introduction}

Laminopathies, a group of inherited human diseases that affect mainly tissues of mesenchymal origin, are associated with structural/functional defect of genes that encode the nuclear envelope proteins. The main component of nuclear lamina, located under the inner nuclear membrane, is lamin $\mathrm{A} / \mathrm{C}$, encoded by the LMNA gene. Mutations in the LMNA gene cause a number of clinical phenotypes, including muscular, peripheral neurogenic, lipodystrophies and premature ageing syndromes (Worman and Bonne 2007). Laminopathies may present as separate disease or as overlapping syndromes; however, clear phenotype-genotype correlations have been difficult to establish. The meta-analysis of Carboni et al. (2013) shows that heterozygous missense mutations spreading throughout the LMNA gene are responsible for the majority of overlapping laminopathies. Mutated lamin $\mathrm{A} / \mathrm{C}$ acts in a dominant-negative way on normal protein translated on the healthy allele, altering its stability and interaction with partner proteins. Here, we present a patient with complex clinical syndrome characterized by dilated cardiomyopathy, limbgirdle dystrophy and partial lipodystrophy with an inflammatory response in the muscle and endomyocardial biopsy specimens. 

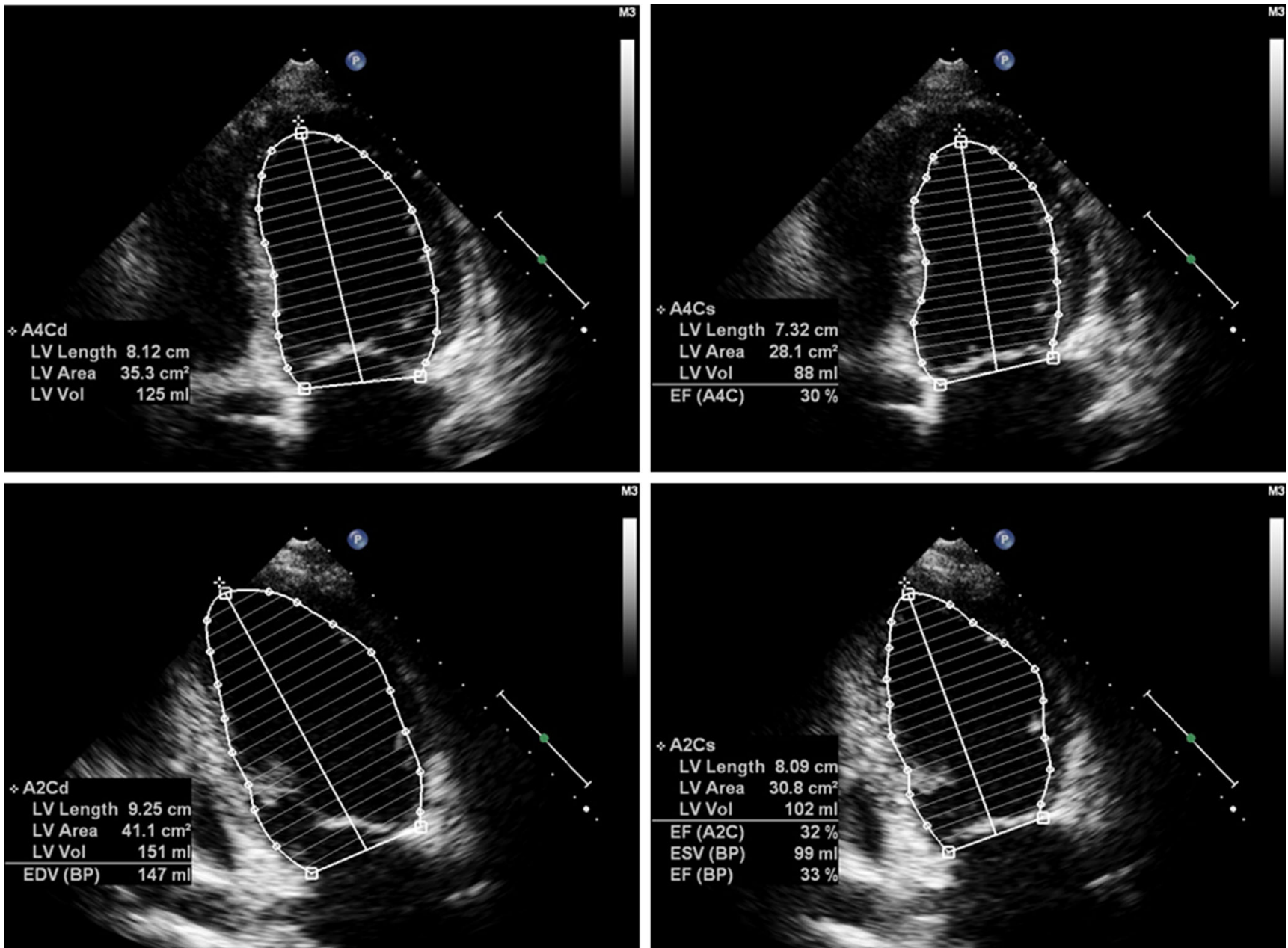

Fig. 1 Restrictive cardiomyopathy on echocardiogram: note significantly decreased ejection fraction (EF) of $33 \%$, increased end-systolic volume (ESV) of $99 \mathrm{ml}$ (normal <50 ml) and end-diastolic volume (EDV) of $147 \mathrm{ml}$ (normal <120 ml)

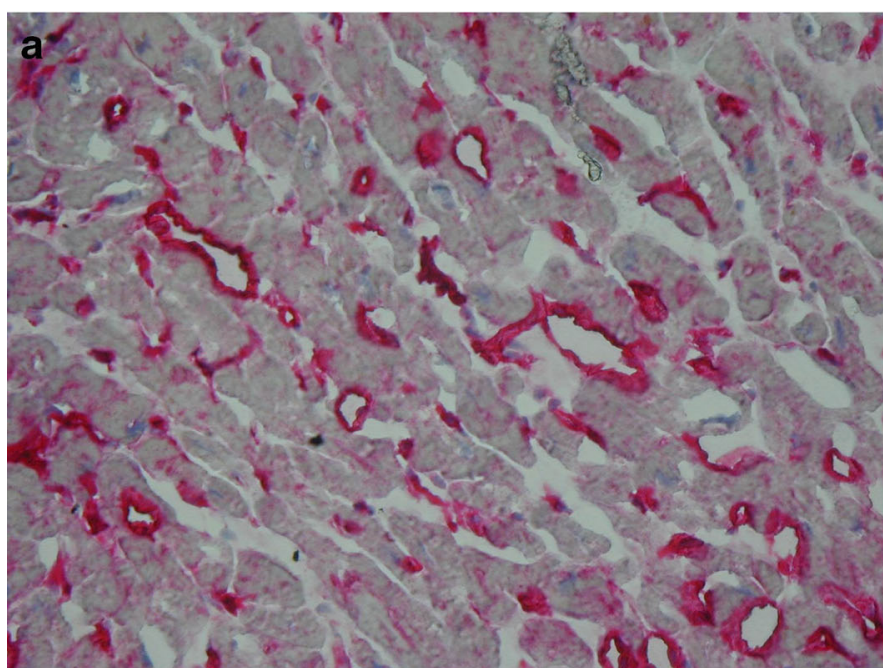

Fig. 2 a Strong expression ( $\mathrm{IR}=2+$ ) of HLA-ABC antigens by microvascular endothelium denoted by the red staining (original magnification $\times 400 \times)$. b Histopathological evaluation of left $\mathrm{m}$. pectoralis major:

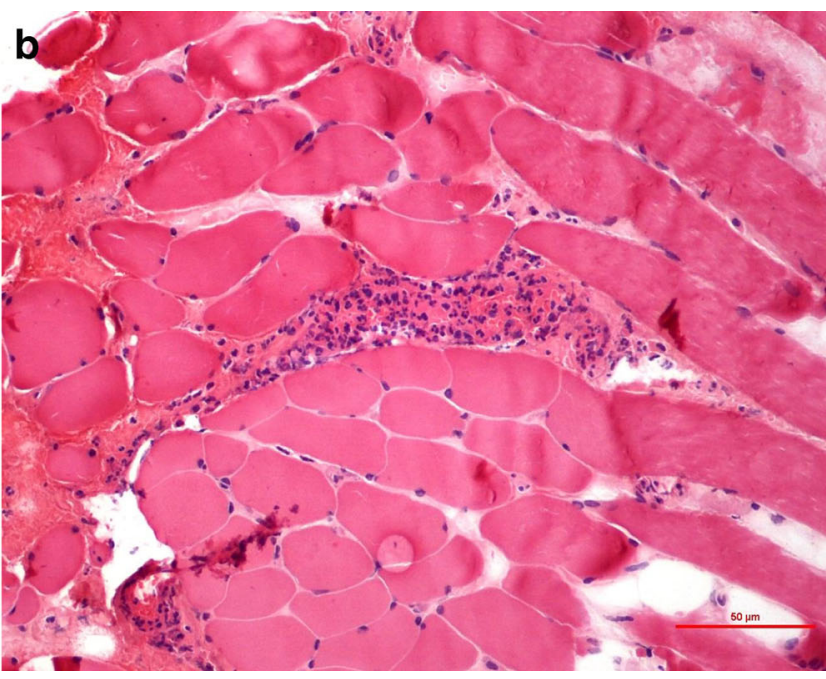

focally distributed inflammatory infiltrations, localized mainly in the perivascular area (original magnification $\times 400 \times$ ) 


\section{Case study}

A 35-year-old Caucasian female with overlapping laminopathy was found to carry a novel sporadic heterozygous mutation c.1001_1003delGCC (p.Ser334del) of the LMNA gene. Although systolic dysfunction was dominated in the clinical presentation, some additional findings helped to establish the final diagnosis of laminopathy. First clinical symptoms such as dyspnea, peripheral edema, palpitations and general weakness occurred at the age of 32 years, suggesting myocardial disease. Electrocardiography revealed atrial fibrillation/flutter and supraventricular tachycardia (SVT) with artio-ventricular block (AVB) 2:1. Echocardiography examination revealed significant systolic dysfunction with left ventricular ejection fraction (EF) of $30 \%$ and pericardial effusion (Fig. 1). The initially considered diagnosis was postinflammatory dilated cardiomyopathy. For this reason, right ventricular endomyocardial biopsy was performed. Histochemistry excluded acute myocarditis based on the Dallas criteria but showed moderate interstitial fibrosis in the biopsy specimens (Masson's trichrome stain). Immunohistochemistry revealed strong endothelial expression of HLA classes I and II antigens with concomitant de novo induction of class I on cardiomyocytes (Fig. 2a). At the cardiology clinic, the patient had moderate symptoms of heart failure [New York Heart Association (NYHA) classes II and III], which was stable under oral treatment. The serum markers of myocardial necrosis were negative. The treatment included electric cardioversion (100 J), which allowed temporary restoration of sinus rhythm with AVB 2:1, followed by SVT with AVB 2:1 and 3:1. During the subsequent 2 years, recurrent atrial fibrillation/flutter were observed, which proved to be resistant to electric cardioversion $(200 \mathrm{~J})$. In addition, ventricular arrhythmia (pairs, triplets) was registered.

Follow-up physical examinations revealed slowly progressing weakness and atrophy of limb-girdle muscles, noted at first during adolescence, absence of knee reflexes, rigid spine with mild hyperlordosis and slight elbow and knee contractures. Electromyography done twice at the ages of 20 years and 34 years did not show myopathic pattern; however, histopathological examination of muscle (m. pectoralis major) revealed variability of fiber diameter and focally distributed inflammatory infiltrations (Fig. 2b). Medical history review of infertility, amenorrhea and excision of bilateral endometrial ovarian cysts at age 32 years, as well as prominent loss of subcutaneous fat on arms and legs, might be consistent with partial lipodystrophy features (Fig. 3). Glucose and lipids were within the normal ranges. All those findings made a potential background of overlapping laminopathy very probable.

Once written informed consent was received, we performed genetic analysis. The study was approved by the local

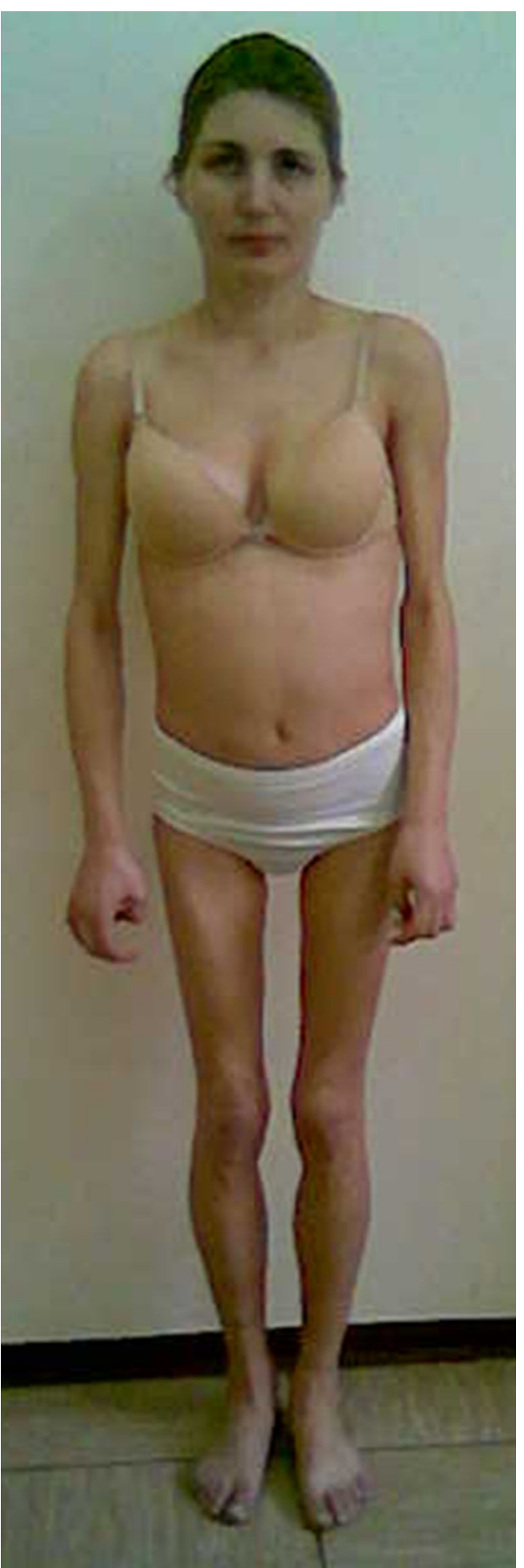

Fig. 3 A 38-year-old female: note atrophy of proximal muscles, slight elbow contractures and hyperlordosis and loss of subcutaneous fat on arms and legs

Bioethical Committee. Genomic DNA was extracted from peripheral blood lymphocytes using the salting-out procedure. All 12 exons of LMNA and exon-intron junctions were amplified by polymerase chain reaction (PCR), sequenced using 
Fig. 4 Direct sequencing showing heterozygous in-frame deletion c.1001_1003delGCC (p.Ser334del) in exon 6 of the LMNA gene

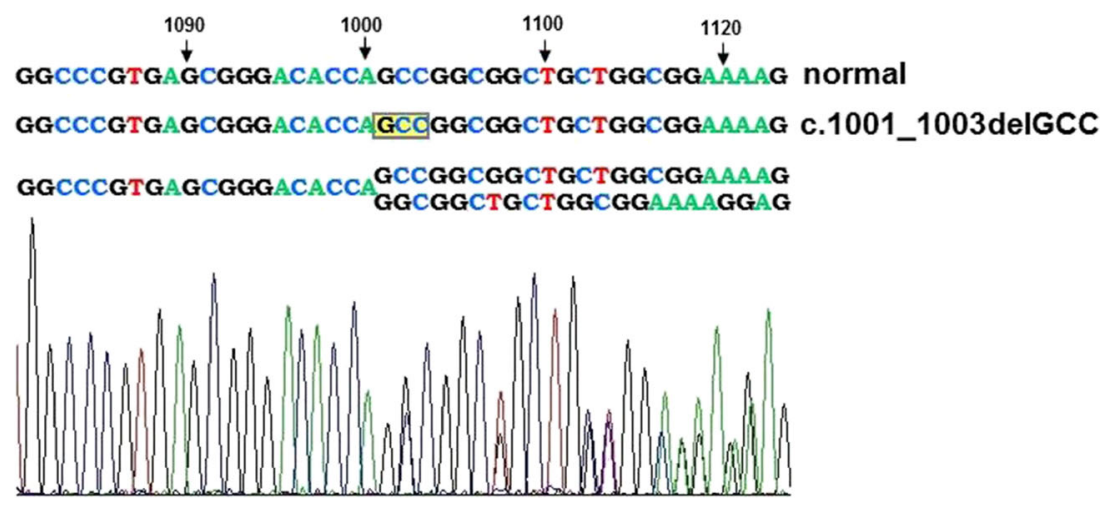

the BigDye Terminator Sequencing Ready Reaction Kit (Applied Biosystems) and analysed on an ABI PRISM 373 fluorescent DNA sequencer (Applied Biosystems). Primer sequences and PCR protocols are available upon request. In fact, direct DNA sequencing of 12 exons and exon-intron boundaries of the LMNA gene revealed a heterozygous in-frame deletion c.1001_1003delGCC (p.Ser334del) of the LMNA gene (Fig. 4).

A final diagnosis of laminopathy guided follow-up cardiological therapy. Because of the high risk of sudden cardiac death due to significant systolic dysfunction and mutation in the LMNA gene, a cardioverter-defibrillator (Biotronik Setrox S 53) was implanted. However, despite optimal therapeutic strategy, progression of heart failure had been observed. At the age of 35 years, the patient underwent heart transplantation and, 5 years after surgery, its effect remained satisfactory. Skeletal muscle disease is gradually progressing, leading to marked muscle atrophy with concomitant quite well preserved strength. In addition to fat loss from the extremities, accumulation of subcutaneous fat on the neck, face and trunk, typical for partial lipodystrophy, is seen. Regular menses returned after transplantation, so it seems that amenorrhea might be partially associated with poor nutrition status in the period of heart failure.

\section{Discussion}

There are several reports of laminopathies characterized by complex phenotype, composed of lipodystrophy, cardiac disease and muscular dystrophy. Two of three patients described in the paper of van der Kooi et al. (2002), bearing missense mutations in exon 9 of the LMNA gene, had features of partial lipodystrophy and muscle weakness, spine rigidity and joint contractures observed since adolescence. In contrast to the patient described here, their cardiac presentation included atrial fibrillation without symptoms of cardiomyopathy. The third patient, having mutation in exon 1 of the LMNA gene, had partial lipodystrophy and congestive heart failure with conduction defect, but the first signs of cardiac disease occurred later than in our patient, i.e. in the fifth decade of life. In contrast to our patient, partial lipodystrophy was accompanied by hypertriglyceridemia and diabetes mellitus, and skeletal muscles were not affected. Two other families with familial partial lipodystrophy (FPLD) and cardiomyopathy with conduction system defects and proximal muscle weakness, caused by missense mutations in exon 1 of the LMNA gene, were described by Garg et al. (2002), although cardiac manifestation was not predominant in these cases.

The LMNA variant c.1001_1003delGCC (p.Ser334del) in the patient presented here was reported in the meta-analysis of Carboni et al. (2013), although without a detailed description of its clinical consequences. It is localized in exon 6 of the LMNA gene and it results in the deletion of serine in position 334 of the lamin $\mathrm{A} / \mathrm{C}$. This residue is located in the central alpha-helical rod domain of the protein and it is responsible for lamin A homodimer formation. As interhelical salt bridges are between residues p.Glu330 and p.Arg331 and between p.Lys331 and p.Glu342, we might presume that the deletion of Ser334 impaired stereochemical interaction of the protein (Strelkov et al. 2004). This pathomechanism of laminopathy was postulated by other authors in patients with missense or frameshift mutations in the $2 \mathrm{~B}$ coil domain of lamin A/C (Benedetti et al. 2007; Volpi et al. 2010). Deletions in the LMNA gene are rare and responsible for only $6 \%$ of known mutations of this gene. There was a report of missense point mutations in position 1003 , i.e. c. $1003 \mathrm{C}>\mathrm{T}$ (p.Arg335Trp), which was associated with complex phenotype, including muscle weakness, atrial fibrillation, hypertriglyceridemia and acro-osteolysis, presented by Drouin et al. during the 54th Annual Meeting of the American Society of Human Genetics (ASHG) in 2004. Other reported mutations located in close vicinity to nucleotide position 1000 of the LMNA gene have been shown to result in cardiomyopathy with conduction disturbances and, finally, sudden cardiac death, despite the implantation of a cardioverter-defibrillator (c.992G >A, p.Arg331Gln) (Møller et al. 2009) and in Emery-Dreifuss muscular dystrophy (EDMD) with early cardiac onset (c.1007G>A, p.Arg336Gln) (Raffaele Di Barletta et al. 2000; Sanna et al. 2003). Complex phenotype comprised of limb-girdle muscular dystrophy (LGMD) with atrial fibrillation and tachy-brady syndrome, 
features of FPLD, short stature and facial dysmorphy was described by Mercuri in a patient with mutation p.E358K in exon 6 of the LMNA gene. The same mutation was responsible for the EDMD phenotype in four other patients. Interestingly, in one of them, mild up-regulation of HLA class I on the sarcolemma of mature fibers was found in muscle biopsy specimen, which might be suggestive for inflammatory myopathy. In our patient, similar up-regulation and cardiomyocyte induction of HLA class I antigens were observed. In addition to the variability of fiber diameter in the skeletal muscle biopsy consistent with dystrophic changes, focally distributed inflammatory infiltrations, localized mainly in the perivascular area, were seen. A similar observation has already been made by Komaki et al. (2011), who described perivascular cuffing and/or endomysial/perimysial lymphocyte infiltration in patients with $L M N A$ mutations, initially presenting signs of infantile onset inflammatory myopathy and then developed joint contractures and/or cardiac involvement. Hence, it is suggested that inflammation might be an additional pathological process in affected cardiac and skeletal muscles in laminopathy. Significant interstitial myocardial fibrosis, which was seen in our patient in the period of fast progressing heart failure, is consistent with observations of other authors in patients with cardiomyopathy associated with defects of the LMNA gene (Fatkin et al. 1999; van Tintelen et al. 2007; Holmström et al. 2011). Replacement of cardiomyocytes by connective tissue, especially of the basal septum, leads to conduction abnormalities and atrial and ventricular arrhythmia, which is usually the first manifestation of cardiac disease in laminopathy.

In conclusion, upon the rapid progression of heart failure with arrhythmias or conduction defects, accompanied by muscle atrophy and lipodystrophy, the genetic disease should be taken into consideration, even in negative familiar history. Myocardial fibrosis or unspecific inflammatory signs in microscopic examination of heart or skeletal muscle might further justify screening of the $L M N A$ gene.

\section{Compliance with ethical standards}

Conflict of interest The authors declare that they have no financial or other conflicts of interest in relation to this research and its publication.

Ethical issues All procedures were in accordance with the standards of the bioethical committee and the Declaration of Helsinki.

Open Access This article is distributed under the terms of the Creative Commons Attribution 4.0 International License (http:// creativecommons.org/licenses/by/4.0/), which permits unrestricted use, distribution, and reproduction in any medium, provided you give appropriate credit to the original author(s) and the source, provide a link to the Creative Commons license, and indicate if changes were made.

\section{References}

Benedetti S, Menditto I, Degano M, Rodolico C, Merlini L, D’Amico A et al (2007) Phenotypic clustering of lamin A/C mutations in neuromuscular patients. Neurology 69:1285-1292

Carboni N, Politano L, Floris M, Mateddu A, Solla E, Olla S et al (2013) Overlapping syndromes in laminopathies: a meta-analysis of the reported literature. Acta Myol 32:7-17

Fatkin D, MacRae C, Sasaki T, Wolff MR, Porcu M, Frenneaux M et al (1999) Missense mutations in the rod domain of the lamin A/C gene as causes of dilated cardiomyopathy and conduction-system disease. N Engl J Med 341:1715-1724

Garg A, Speckman RA, Bowcock AM (2002) Multisystem dystrophy syndrome due to novel missense mutations in the amino-terminal head and alpha-helical rod domains of the lamin $\mathrm{A} / \mathrm{C}$ gene. Am J Med 112:549-555

Holmström M, Kivistö S, Heliö T, Jurkko R, Kaartinen M, Antila M et al (2011) Late gadolinium enhanced cardiovascular magnetic resonance of lamin $\mathrm{A} / \mathrm{C}$ gene mutation related dilated cardiomyopathy. J Cardiovasc Magn Reson 13:30

Komaki H, Hayashi YK, Tsuburaya R, Sugie K, Kato M, Nagai T et al (2011) Inflammatory changes in infantile-onset LMNA-associated myopathy. Neuromuscul Disord 21:563-568

Møller DV, Pham TT, Gustafsson F, Hedley P, Ersbøll MK, Bundgaard H et al (2009) The role of Lamin A/C mutations in Danish patients with idiopathic dilated cardiomyopathy. Eur J Heart Fail 11:10311035

Raffaele Di Barletta M, Ricci E, Galluzzi G, Tonali P, Mora M, Morandi L et al (2000) Different mutations in the LMNA gene cause autosomal dominant and autosomal recessive Emery-Dreifuss muscular dystrophy. Am J Hum Genet 66:1407-1412

Sanna T, Dello Russo A, Toniolo D, Vytopil M, Pelargonio G, De Martino G et al (2003) Cardiac features of Emery-Dreifuss muscular dystrophy caused by lamin A/C gene mutations. Eur Heart J 24: 2227-2236

Strelkov SV, Schumacher J, Burkhard P, Aebi U, Herrmann H (2004) Crystal structure of the human lamin A coil 2B dimer: implications for the head-to-tail association of nuclear lamins. Mol Biol 343: $1067-1080$

van der Kooi AJ, Bonne G, Eymard B, Duboc D, Talim B, Van der Valk $\mathrm{M}$ et al (2002) Lamin A/C mutations with lipodystrophy, cardiac abnormalities, and muscular dystrophy. Neurology 59:620-623

van Tintelen JP, Tio RA, Kerstjens-Frederikse WS, van Berlo JH, Boven LG, Suurmeijer AJ et al (2007) Severe myocardial fibrosis caused by a deletion of the $5^{\prime}$ end of the lamin $\mathrm{A} / \mathrm{C}$ gene. J Am Coll Cardiol 49:2430-1439

Volpi L, Ricci G, Passino C, Di Pierri E, Alì G, Maccherini M et al (2010) Prevalent cardiac phenotype resulting in heart transplantation in a novel LMNA gene duplication. Neuromuscular Disord 20:512-516

Worman HJ, Bonne G (2007) "Laminopathies": a wide spectrum of human diseases. Exp Cell Res 313:2121-2133 